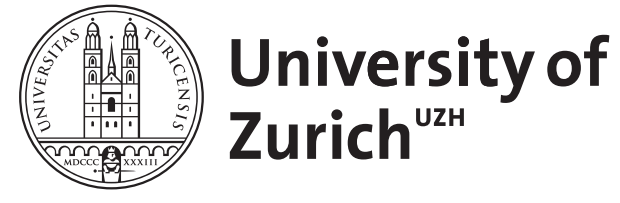

Was (wenn überhaupt etwas) ist falsch an der Perspektive?

Laleg, Dominique

DOI: https://doi.org/10.28937/1000108214

Posted at the Zurich Open Repository and Archive, University of Zurich

ZORA URL: https://doi.org/10.5167/uzh-205261

Journal Article

Accepted Version

Originally published at:

Laleg, Dominique (2019). Was (wenn überhaupt etwas) ist falsch an der Perspektive? Zeitschrift für Ästhetik und allgemeine Kunstwissenschaft, 63(2):247-266.

DOI: https://doi.org/10.28937/1000108214 


\section{WAS (WENN ÜBERHAUPT ETWAS) IST FALSCH AN DER PERSPEKTIVE?}

\section{Die Perspektive relativieren}

Die Physiologie des 19. Jahrhunderts bildet die erste gravierende Wende in der modernen Bedeutungsgeschichte der Perspektive. Ihre Erkenntnisse und nicht zuletzt die auf der Basis dieser Erkenntnisse entwickelten Apparate verändern das Verständnis der menschlichen Wahrnehmung maßgebend. Diese Veränderung wird in der Wissenschaftsgeschichte der foucaultschen Tradition prominent als Bruch besprochen und markiert so gesehen auch eine Zäsur in der westlichen Bildgeschichte. ${ }^{1}$ Die zunehmenden Erkenntnisse und Bedeutung der Physiologie als Bruch mit den Modellen der klassischen Repräsentation der Wirklichkeit zu besprechen, heißt auch die Physiologie als Kritik an der Perspektive zu verstehen. Diese Kritik beruht dann wesentlich in der Falsifizierung der perspektivischen Darstellungsweise und zwar durch kritische Vergleiche mit biologischen Bedingungen des menschlichen Sehapparates. Ein zentraler Kritikpunkt, den die Physiologie dabei gegen die Perspektive richtet, lautet: Das perspektivisch konstruierte Bild ist ungenügend bzw. ungenau, weil es sich bei seinem angeblichen Vorbild und Analogon, dem sogenannten Netzhaut- oder auch Retinabild, um eine Projektion auf eine konkave Fläche handelt und nicht, wie es in der üblichen perspektivischen Konstruktion der Fall ist, um eine planare Fläche. Daraus folgt, dass das sogenannte Netzhautbild Kurvaturen aufweist, die das perspektivisch konstruierte Bild ignoriert. Eine viel rezipierte Studie zu diesem Problem bieten die Untersuchungen Guido Haucks, der mit dem Konzept der kurvilinearen Perspektive ein Mittel entwickelt hat, das die adäquate Übertragung biologischer Sehbedingungen auf die Formgebung des Bildes verspricht (Abb. 1). ${ }^{2}$ Obschon die Netzhautbild-Kritik des perspektivischen Bildes eine lange Geschichte besitzt, die bis zu den Ursprüngen der Perspektive zurückführt, ${ }^{3}$ ist für unser Interesse vor allem entscheidend, dass sie noch in jenen Überlegungen eine zentrale Rolle spielt, die bis heute das Fundament dessen bilden, was man als moderne Perspektivkritik bezeichnen kann.

\footnotetext{
$1 \quad$ Dazu Jonathan Crary: Techniken des Betrachters - Sehen und Moderne im 19. Jahrhundert, Dresden/Basel 1996; sowie Robert Brain: The Pulse of Modernism-Physiological Aesthetics in Fin-de-Siècle Europe, Seattle/London 2015.

2 Guido Hauck: Die subjektive Perspektive und die horizontalen Curvaturen des dorischen Styls, Stuttgart 1879.

3 Siehe etwa Leonardo da Vinci: Sämtliche Gemälde und die Schriften zur Malerei, hg. von André Chastel, München 1990, $241 \mathrm{f}$.
} 
Erwin Panofsky wird in seinem ursprünglich als Vortrag konzipierten Text Die Perspektive als ssymbolische Form die physiologische Argumentation Haucks aufnehmen, fortschreiben und damit eine theoretische Grundlage der modernen Perspektivtheorie formulieren. ${ }^{4}$ Panofskys Ergänzungen der Netzhautbild-Kritik sind untrennbar mit seinem Ort in der Geschichte des Denkens verbunden, der sich als psychologistisch, historisierend und zugleich als neukantianisch beschreiben lässt. Auch Panofsky gilt ein vermeintlich natürliches Sehen als absolute Referenz, mit der sich die perspektivische Darstellungsform messen muss. Doch obschon sich Panofsky explizit auf Hauck beruft, spielt für ihn nicht einzig die Anatomie des Auges, sondern auch die psychologische Interpretation der bewussten Wahrnehmung eine zentrale Rolle bei der Entstehung des subjektiven Seheindrucks. Die Zusammensetzung dieser beiden Bedingungen führt dazu, dass Panofsky vom "psychophysiologischen Raum« der Wahrnehmung spricht. ${ }^{5}$ Diese symptomatischen Begrifflichkeiten verweisen nicht nur auf die hegemonialen Modelle der Wahrnehmung des ausgehenden 19. und frühen 20. Jahrhunderts, sondern auch auf jenen Parameter, an dem sich die historische Bedeutung der Renaissanceperspektive für Panofsky ermessen lässt: der Raum. Panofsky skizziert entlang mehrerer Epochen eine dialektische Geschichte des Bildraums, in welcher er zugleich die abendländische Geistesgeschichte erkennen will. Eine Raumanschauung, so seine Hypothese, hängt stets mit einer Weltanschauung zusammen. Die Problematik und Undeutlichkeit dieses Zusammenhangs, den Panofsky mit einem Hinweis auf Ernst Cassirers Philosophie der symbolischen Formen zu klären glaubt - ihn damit aber eher verunklärt -, können hier nicht genauer behandelt werden. ${ }^{6}$ Festgehalten werden soll aber, dass die Perspektive als Moment einer dialektischen Ideengeschichte begriffen wird, die unter dem Einfluss physiologischer sowie psychologischer Bedingungen zum Gegenstand einer relativierenden und mitunter historisierenden Kritik wird. Demnach ist die perspektivische Bildform nicht nur dahingehend zu kritisieren, dass sie der erlebten visuellen Wahrnehmung nicht gerecht wird, sie ist zudem bloß eine historisch bedingte Weise der Darstellung unter vielen.

Die physiologische sowie die historisierende Kritik an der perspektivischen Form, die sich in Panofskys Aufsatz verbinden, teilen die Gemeinsamkeit, dass sie die Perspektive zum bloßen Objekt der Kritik reduzieren. Die physiologische Position formuliert ihre Kritik scheinbar im Ausgang von einem der Perspektive prinzipiell externen Standpunkt, dem der Physiologie. Sie scheint insofern legitim, als dass sie diesen Standpunkt deutlich macht und aus

\footnotetext{
4 Erwin Panofsky: Die Perspektive als »symbolische Form« (1927), in: Aufsätze zu Grundfragen der Kunstwissenschaft, Berlin 1974, 99-167.

$5 \quad$ Ebd., $101 \mathrm{f}$.

6 Dazu Emmanuel Alloa: Ist die Perspektive eine symbolische Form?, in: Perspektive - Die Spaltung der Standpunkte, hg. von Gertrud Koch, München 2010, 13-27.
} 
der damit erzeugten Distanz Kritik übt. Ob sie diese Distanz tatsächlich einhält, wird jedoch fraglich, wenn man ihre Prämissen genauer analysiert, die möglicherweise ihrerseits mit der Perspektive verquickt sind; bedenkt man, dass die Argumentation der Netzhautbild-Kritik wesentlich darauf beruht, dass das Perspektivbild dem Netzhautbild nicht in korrekter Weise entspricht. Dies setzt voraus, dass es sich bei der Ansammlung gebündelten Lichts auf der Netzhaut überhaupt um so etwas wie ein Bild handelt, was wiederum nicht nur den Gegenstandsbereich der Physiologie und Optik übersteigt - denn diese kennen überhaupt keine Bilder im engeren Sinn -, sondern zudem voraussetzt, dass der Sehvorgang des Auges ein Vorgang von bzw. mit Bildern ist. Nun fällt die früheste bildtheoretische Konzeption des Sehvorgangs als einem quasi-piktoralen Prozess und die damit überhaupt ermöglichte Idee einer Äquivalenz zwischen Sehbild und artifiziellem Bild aber nicht zufällig mit dem theoretischen Gründungsakt der Perspektive im 15. Jahrhundert zusammen. Albertis De pictura (1535/36) ist eines der ersten uns bekannten Dokumente, das die Vorstellung formuliert, das Auge sehe in Bildern, und zwar in unmittelbarem und explizitem Zusammenhang mit der perspektivischen Konstruktion. ${ }^{7}$ Dagegen sind die für Alberti zugrunde liegenden Traktate der Optik (allen voran Euklid, Ptolomäus und Alhazen) keine expliziten Theorien des Bildes, sondern Theorien der visuellen Wahrnehmung. Diese antiken bzw. mittelalterlichen Sehtheorien begreifen das finale Gelingen des Sehvorgangs und damit die Bedingung der Bildwerdung stets in Abhängigkeit von der vielschichtigen, ambivalenten und aktiven Imagination als einem Vermögen, das in der Subjektphilosophie als Einbildungskraft bezeichnet wird und die anatomischen Bedingungen des Auges bei weitem überschreitet. ${ }^{8}$ Wenn aber Albertis Theorie der Perspektive zu den ersten gehört, die überhaupt so etwas wie ein Sehbild konzipiert - um dann das perspektivisch konstruierte Bild als dessen Äquivalent zu bestimmen -, so dreht sich die Argumentation der modernen Netzhautbild-Kritik im Kreis. Denn in diesem Fall formuliert sie ihren zentralen Kritikpunkt an der Perspektive ausgerechnet in Berufung auf das Paradigma der Perspektive - eine seltsame Sachlage, die für die Beziehung von Kritik und Perspektive möglicherweise symptomatisch ist.

Mit Panofskys Kritik scheint sich die Sache zu verkomplizieren, denn er erkennt jene in seiner Deutung >psychologischen< Prozesse und Voraussetzungen an, die sich zwischen den affizierenden Lichtstrahlen auf der Netzhaut und der bewussten Wahrnehmung abspielen. Die für unsere Fragestellung relevanten Überlegungen liegen daher auch nicht in Panofskys

\footnotetext{
7 Leon Battista Alberti: De pictura, in: Das Standbild-Die Malkunst-Grundlagen der Malerei (1435), hg. von Oskar Bätschmann und Christoph Schäublin, Darmstadt 2000, 194-335.

Dazu auch Gérard Simon: Archéologie de la vision, Paris 2003; Le regard, l'être et l'apparance dans l'Optique de l'Antiquité, Paris 1988.
} 
Konzept der psychophysiologischen Wahrnehmung, sondern in der Makrostruktur seiner Argumentation. Panofskys Schlussüberlegungen, die er im vierten und letzten Teil seines Textes formuliert, zeigen dass er die Renaissanceperspektive als systematische Verschränkung des Objektiven mit dem Subjektiven begreift und in ihr daher ein homologes Äquivalent der Transzendentalphilosophie erkennt. Der perspektivisch konstruierte Bildraum wird hier als eine symmetrische Synthese von rationalem und empirischem Raum verstanden, eine Art systematisches Konglomerat von theoretischem Idealraum und subjektiv erschlossenem Wahrnehmungsraum.

So gesehen besitzt die Perspektive für Panofsky im doppelten Sinn Modellcharakter. Denn perspektivische Bilder sind modellhafte Darstellungen der Wahrnehmungsbilder und -räume, insofern sie diese in abstrahierter Weise wiedergeben. Die Perspektive ist aber auch ein epistemologisches Modell, da der durch sie erzeugte Bildraum als Analogon der subjektiven Erscheinungswelt begriffen wird. Diese zweite Analogie fußt auf der kantianischen Prämisse, dass die phänomenale Welt `bloße Vorstellungく eines Subjekts ist und dennoch der kategorialen Regelhaftigkeit des Verstandes folgt, mithin in ihrer Erscheinungsweise berechenbar ist. Dieses transzendentalphilosophische Prinzip einer Quasiverschränkung von Subjektivität und Objektivität sieht Panofsky in der Renaissanceperspektive vorweggenommen.

Blickt man von hier auf die Argumentation des gesamten Texts zurück, stellt sich die Frage, ob Panofskys ideengeschichtlicher Begriff der $>$ Weltanschauung $<$, mit dem er die Perspektive einer historisierenden und damit relativierenden Kritik unterwirft, nicht eben diesem epistemologischen Modell des Kantianismus verpflichtet ist - ob die Idee von so etwas wie >Weltanschauung « erst auf der Basis jener spezifischen Subjekt-Objekt-Konstellation sowie einer damit einhergehenden epistemologischen Rehabilitation der Anschauung durch Rationalisierung möglich wird. Dies würde jedoch bedeuten, dass der gesamte konzeptuelle Überbau des Textes, der eine wechselseitige Beziehung zwischen bildlicher Darstellung und (Welt-)Anschauung postuliert - eine Beziehung, die Panofsky als bildgeschichtliche Konstante voraussetzt -, letztlich in der Perspektive als einem epistemologischen Modell gründet. Die historisierende Kritik der Perspektive, die auf einer radikalen Relativierung derselben beruht, verliert jedoch ihre Kraft, um nicht zu sagen: ihren Sinn, wenn deutlich wird, dass man für das vorperspektivische Zeitalter keine dialektische Geschichte der Perspektive schreiben kann, ohne sich auf die Perspektive als quasi-überhistorischen Referenzpunkt zu beziehen.

Es wird deutlich, dass sowohl die physiologische als auch die historisierende Kritik an der perspektivischen Formgebung einer Problematik aufsitzt, die mit der Perspektive und ihrer 
komplexen Bedeutung unmittelbar zusammenhängt - und zwar nicht bloß für die Form piktoraler Darstellung, sondern ebenso für die Form des Denkens. Denn die wissenschaftliche Kritik der Perspektive scheint hier selbst mit den Mitteln der Perspektive zu denken, indem sie ihre Prämissen voraussetzt, ohne dies zu merken. So gesehen ist dieses Denken von der Perspektive selbst informiert, was letztlich darauf hinweist, dass Kritisiertes und Kritisierendes miteinander verwoben sind. Doch worauf verweist diese Verwicklung und damit die Problematik, dass die Kritik der Perspektive dieser selbst nicht entkommt? Deutet der Umstand, dass ausgerechnet die prominentesten Perspektivkritiker der Moderne ihre Kritik an der Perspektive zugleich mit der Perspektive formulieren, möglicherweise auf ein der perspektivischen Form innewohnendes kritisches Vermögen?

\section{Modernistische Selbstkritik}

Will man die schwierige Beziehung der Moderne zur Perspektive besser verstehen, kommt man nicht umhin, diese Beziehung im Hinblick auf die Bedeutung von >Kritikı zu befragen. Im Zusammenhang mit der Entwicklung der bildenden Künste im 20. Jahrhunderts bildet die Kunsttheorie Clement Greenbergs dabei ein besonders geeignetes Objekt der Analyse, denn sie verbindet drei Elemente, die für die hier vorliegende Fragestellung relevant sind: Erstens formiert Greenbergs Theorie, die in der Mitte des 20. Jahrhunderts entsteht, die malereigeschichtlichen Entwicklungen des 19. und frühen 20. Jahrhunderts zu einem einflussreichen Narrativ, das vor allem für die spätmodernistische Malereiproduktion und -rezeption maßgebend sein wird; zweitens erlaubt der formalistische Charakter seiner Theorie die Rolle der Perspektive zu bestimmen; drittens sind diese historisch bedeutende Narrativbildung und ihre formalistischen Kriterien auf dezidierte Weise mit einer Konzeption von Kritik verknüpft - eine Kritik, die auch ausdrücklich eine Kritik der perspektivischen Formgebung ist und das moderne Verständnis der Perspektive entscheidend prägen wird.

Greenberg entwickelt ab Mitte des 20. Jahrhunderts eine Gruppe von Kriterien, die für den Fortgang modernistischer Formparadigmen so einflussreich wie problematisch sein wird. ${ }^{9}$ Diese Kriterien lassen sich auf drei grundlegende Momente reduzieren, die sich als drei zusammenhängende Hypothesen erweisen.

\footnotetext{
9 Clement Greenberg: Zu einem neuen Laokoon (1940), in: Die Essenz der Moderne-Ausgewählte Essays und Kritiken, Hamburg 2009, 56-81.
} 
Erstens das Kriterium kritischer Reflexion, das Greenberg u.a. im Rekurs auf Kants kritische Philosophie entwickelt. Das Wesen des Modernismus liegt demnach in seinem kritischen Selbstbezug, d.h., indem die Methode eines bestimmten Gegenstandsbereichs in kritischer Weise auf sich selbst angewendet wird, um ihre eigene Legitimität zu prüfen bzw. zu stärken. Für den Gegenstandsbereich der Kunst bedeutet dies zunächst, dass die künstlerischen Verfahrensweisen selbst zum Gegenstand der Kunst avancieren. ${ }^{10}$

Von dieser zunächst systematischen These geht Greenberg zu seinem zweiten und dabei formalistischen Kriterium über, nämlich der Bestimmung dessen, was künstlerische Verfahrensweisen sind. Denn diese, so wird im Text Avantgarde und Kitsch erläutert, ${ }^{11}$ sind nichts anderes als die gestalterischen Verfahrensweisen des jeweiligen »Mediums«, d.h. im Fall der bildenden Künste das »Finden und Ordnen von Räumen, Flächen, Formen, Farben etc. «. ${ }^{12}$ Kritische Selbstreflexion kommt in der Praxis der bildenden Kunst also dadurch zum Tragen, dass diese ein »Nachahmen des Nachahmens« vollzieht und damit ihre eigenen Mittel, die als Gestaltungsmittel bestimmt werden, befragt und exponiert. ${ }^{13}$

Bis zu diesem Punkt müsste die Perspektive kein prinzipielles Problem für die avantgardistische Praxis werden, denn es ist nicht einsichtig, weshalb ein kritischer Selbstbezug sowie eine Reflexion ihrer gestalterischen Verfahrensweisen prinzipiell nicht möglich sein sollen. Mit Greenbergs dritter und materialistischer Hypothese ändert sich dies jedoch, und zwar indem er seine Bestimmung des Mediums weiter zuspitzt. Dieses wird nicht mehr bloß mit den gestalterischen Verfahrensweisen im weitesten Sinne in Verbindung gebracht, sondern zunehmend an die materiellen Eigenschaften der jeweiligen Gattung gebunden. Für die Malerei bedeutet das schließlich, dass die Materialität des Bildträgers zum virulenten Bestandteil kritischer Reflexivität des Modernismus wird. Greenberg glaubt jene Virulenz in einer »neue[n] Flächigkeit« zu erkennen, die seit Gustave Courbet Einzug in die Malerei gehalten habe und seither eine zunehmende Anerkennung innerhalb der Disziplin gewinne. ${ }^{14}$ Diese historisch zunehmende Anerkennung und Betonung hat gleichsam teleologischen Charakter und erfährt für Greenberg in der abstrakten Malerei und insbesondere in jener der amerikanischen Nachkriegszeit eine Art Höhepunkt.

Dieses dritte Kriterium ist von besonderer Tragweite im Hinblick auf die Perspektive. Denn das malerische Medium wird nicht mehr bloß als Dispositiv bestimmt, bestehend aus den

\footnotetext{
$10 \quad$ Ebd., 267.

11 Clement Greenberg: Avantgarde und Kitsch (1939), in: Die Essenz der Moderne - Ausgewählte Essays und Kritiken, Hamburg 2009, 29-55.

12 Ebd., 35.

13 Ebd., 35.

14 Greenberg: Zu einem neuen Laokoon [Anm. 9], 66.
} 
unterschiedlichsten gestalterischen Verfahren, die in ihrer Art und Anzahl prinzipiell unbegrenzt und beliebig sind, sondern es wird nun mit dem materiellen Substrat des Bildes identifiziert. Die Avantgarde zeichnet sich folglich dadurch aus, dass sie die »Undurchdringlichkeit der Bildoberfläche« anerkennt und dieser dezidiert »materiellen Flächigkeit « Ausdruck verleiht. ${ }^{15}$ Unter dieser Prämisse kommt Greenberg schließlich dazu, die künstlerische Praxis der Avantgarde als Antithese der perspektivischen Formgebung und des durch sie erzeugten illusionistischen Raums folgendermaßen zu beschreiben:

»Die Geschichte der Avantgarde Malerei ist die Geschichte ihrer schrittweisen Anerkennung der Widerständigkeit ihres Mediums, welche hauptsächlich darin besteht, dass die plane Bildfläche den Versuchen widersteht, sie $\mathrm{zu}$ einem realistischen perspektivischen Raum hin zu durchstoßen.$^{16}$

Ohne Greenbergs Konzeption bzw. Narrativ der modernistischen Repräsentationskritik sowie dessen Folgen und Probleme für die Praxis und Theorie der Malerei weiter zu erörtern, lässt sich festhalten, dass auf der Basis der drei geschilderten Hypothesen eine explizite Kritik der Perspektive artikuliert wird. Diese wird für Greenberg zum grundsätzlichen Gegenspieler reflexiver Kritik und damit des Modernismus schlechthin. Dabei unterscheidet sich Greenbergs Kritik der Perspektive vor allem dahingehend von Panofskys Kritik, als dass sie ihre Kriterien nicht auf der Basis einer gelingenden Äquivalenzbeziehung zwischen Perspektivbild und einem vermeintlich natürlichen bzw. erlebten Wahrnehmungsbild entwickelt. Im Gegensatz zu dieser an Veridität interessierten Kritik baut Greenberg auf einer Logik auf, die man als selbstreflexiven Kritizismus beschreiben könnte, und den er als formtheoretisches Paradigma für die Moderne Malerei einsetzt. Dieser Unterschied markiert eine wichtige Entwicklung der späten ästhetischen Moderne, die sich und ihre Paradigmen zunehmend vom Bereich der Wissenschaft abtrennen will. Dabei wird die Perspektive nicht deshalb zum Objekt der Kritik, weil ihre Ergebnisse ungenügende Äquivalente des wissenschaftlich untersuchten Sehvorgangs sind, sondern weil es scheint, als sei das perspektivische Bild nicht mit einem bestimmten Paradigma der Selbstkritik vereinbar. Genau diese Schlussfolgerung aber möchte ich anzweifeln, indem ich die Frage aufwerfe, ob Greenbergs Paradigmen die perspektivische Bildform möglicherweise zu Unrecht als Antithese einer reflexiven Kritik in seinem Sinn 
beurteilt. Natürlich hängt das Schicksal der Perspektive in der Moderne nicht einzig von dieser Beurteilung $a b$, jedoch hat die Tragweite von Greenbergs Modernismus-Konzeption das historische sowie theoretische Verständnis der Perspektive bis in die Gegenwart geprägt. Die Notwendigkeit dieser Beurteilung in Zweifel zu ziehen, bedeutet daher auch, über die Konstruktion eines größeren Narrativs nachzudenken, das unser Bild der Moderne mitbestimmt.

Ich möchte an dieser Stelle die Problemlage, die Greenbergs selbstreflexiver Kritizismus mit sich bringt, verdeutlichen: Ich folge Greenberg zunächst darin, dass der Modernismus als ein wesentlich kritisches, mithin emanzipatorisches Projekt zu verstehen ist, und erkläre mich insoweit einverstanden damit, als dass die Kritik, um die es geht, prinzipiell Selbstkritik ist. Ein starker Begriff von Selbstkritik setzt voraus, dass diese (selbst-)reflexiv verfasst ist. Dazu schreibt Greenberg: »[D]er Modernismus kritisiert von innen heraus und bedient sich dabei der Verfahren eben dessen, was er kritisiert. $\ll^{17}>$ Kritik ८ beschreibt hier nicht einfach die wahllose Beziehung zwischen einem kritisierenden Subjekt und einem kritisierten Objekt. Gemeint ist vielmehr eine selbstreflexive Kritik im engen Sinn: »von innen heraus« bedeutet im Gegensatz $\mathrm{zu}$ einer Kritik von außen, dass das Subjekt und das Objekt der Kritik sich wechselseitig konstituieren. Diese intrinsische, wechselseitige Abhängigkeitsbeziehung führt zur schwierigen Frage, ob und wie eine derartige, dem Kunstwerk immanente Kritik, deren Ziel, mit Fried gesprochen, in der »Selbstbehauptung« des Werks liegen würde, gelingen kann. Es handelt sich hier im Grunde um ein logisches Problem, das sich Greenbergs Modernismus mit der Aneignung des metaphysischen Reflexionsparadigmas einkauft. Dieses Problem liegt darin, eine Erklärung vorzulegen, wie die ernst zu nehmende Kritik eines Systems gelingen soll, wenn die Operatoren der Kritik aus dem zu kritisierenden System selbst stammen. Anders gefragt: Wie soll Kritik möglich sein ohne kritische Distanz, d.h. ohne externen Standpunkt des kritisierenden Subjekts, von dem her sich dieses überhaupt erst kritisch auf ein Objekt beziehen kann?

Wie bereits erläutert wurde, versucht die Netzhautbild-Kritik sowie die historisierende Kritik der Perspektive, aus einer derart transzendenten Position heraus Kritik zu üben. Beide stellen die Legitimität der Perspektive von außen, d.h. von einem jeweils systemexternen Ort, etwa dem der Physiologie, infrage. Sie verstehen die Perspektive dabei nolens volens als bloßes Objekt der Kritik. Wenn das Paradigma des Modernismus jedoch als Reflexion im engeren

17 Clement Greenberg: Modernistische Malerei (1960), in: Die Essenz der Moderne-Ausgewählte Essays und Kritiken, Hamburg 2009, 265-278, hier 266. 
Sinne konzipiert ist, stellt sich die Frage, wie es um die Perspektive als Subjekt der Kritik steht? Der modernistische Diskurs des 20. Jahrhunderts hat diese Frage in einem gewissen Sinn übergangen, indem er den Begriff der Perspektive in Stellung gebracht hat um gewisse Anliegen zu verfolgen. Dabei hat der Konnex zwischen den Begriffen >Selbstreflexion` und >Flächigkeit` dazu geführt, dass die Perspektive als raumbildendes Mittel vom modernistischen Reflexionsparadigma dissoziiert wurde. Die Dichotomie von Fläche und Raum scheint in diesem Zusammenhang einen beinah schicksalshaften Scheideweg eröffnet zu haben, der dazu beigetragen hat, dass die Perspektive nicht als ein potentiell selbstreflexives und damit kritisches bildnerisches Mittel wahrgenommen werden kann.

\section{Anamorphotische Grenzgänge}

Die Perspektive wird im Licht der wissenschaftlichen sowie der ästhetischen Moderne also zur Zielscheibe der Kritik. Es scheint mir nicht unwichtig zu betonen, dass diese Kritik zugleich definitorischen Charakter für die ästhetische Moderne selbst besitzt. Die bis ins 19. Jahrhundert und teilweise länger andauernde hegemoniale Bedeutung der Perspektive und der durch sie gestalteten Bildräumlichkeit, bietet einen hervorragenden Schauplatz, auf dem die künstlerische Moderne ihren kritischen und revolutionären Charakter unter Beweis stellen kann, in dem sie die perspektivische Logik außer Kraft setzt. Im Weiteren wird es darum gehen, dieses populäre Verständnis der Perspektive als bloßes Objekt der Kritik in Zweifel ziehen. Die zentrale Frage lautet dabei: Ist die Perspektive als Modell der bildlichen Formgenese in der Lage, den kritisch-reflexiven Ansprüchen der Moderne gerecht zu werden? Dazu werde ich zunächst einige Überlegungen zur Anamorphose anstellen, die sich mit der Frage beschäftigen, ob und wie in den anamorphotischen Projektionen bereits in der frühen Geschichte der Perspektive eine Kritik angelegt ist und wie man diese Kritik mit dem modernistischen Reflexionsparadigma in Beziehung setzen kann.

Die Anamorphose ist kein Phänomen der Moderne im engeren Sinn, denn wie die Schriften Leonardos beweisen, liegen ihre Ursprünge gerade in der Blütezeit des toskanischen Perspektivismus. Nach einer steilen Karriere als delikates Konstruktionsverfahren, vor allem im 17. Jahrhundert, nimmt sie in der Geschichte der Kunst bald nur noch eine Nebenrolle ein und vermutlich ist Baltrušaitis neben Duchamp der einzige, der ihr im 20. Jahrhundert noch ernsthaftes Interesse schenkt. Für ein adäquates Verständnis der Perspektive und ihrer Kritik ist eine Beschäftigung mit der Anamorphose jedoch unabdingbar, nicht nur weil ihre Phänomene 
belegen, dass die Perspektive schon früh in mannigfaltiger Weise eingesetzt wird, sondern auch weil sie die Bedeutung des modernen Begriffs der >Kritik im Hinblick auf die perspektivische Sichtbarkeit schärfen kann.

Ich möchte zunächst die Phänomene eingrenzen, um die es hier geht. Denn von Interesse sind hier nicht beliebige anamorphotische Phänomene, $\mathrm{zu}$ denen etwa auch die Spiegelanamorphose gehört, sondern lediglich solche, die als linearperspektivische Projektionen konstruiert sind. Es handelt sich in den meisten Fällen um lateral bzw. vertikal verzerrte Bilder, wie sie etwa Jean Francois Niceron in seinem Standardwerk »La perspective curieuse« aus dem Jahr 1638 vorstellt. Er zeigt darin wie der virtuose Perspektivist ein Bild derart konstruiert, dass dieses für den Betrachter lediglich aus einem seitlichen Blickwinkel wahrnehmbar wird, aus der üblichen frontalen Position vor der Bildfläche jedoch bis zur Unkenntlichkeit deformiert erscheint (Abb. 2). Der Reiz solcher anamorphotischer Bilder besteht genau aus diesem Wechselspiel zwischen Formation und Deformation des Sichtbaren, die mit dem Eintreten bzw. Verlassen des korrekten Blickpunktes verbunden sind. Mit dieser perspektivischen Praxis gehen zwei entscheidende Prinzipien einher: Erstens ist der Betrachter relativ zur materiellen Bildfläche als mobiler Betrachter konzipiert. Zweitens ist mit dieser Mobilität die zum Teil kryptische Verzerrung des Bildes prinzipiell auflösbar. Um diesen Umstand besser zu verstehen, muss man sich die Grundsätze der perspektivischen Formgebung in Erinnerung rufen.

Das übliche perspektivische Darstellungsverfahren basiert auf der Idee, dass sich die Projektionsebene der perspektivischen Konstruktion parallel zur empirischen (und zumeist planaren) Bildfläche und dabei im 90-Winkel zur zentralen Blickachse befindet (Abb. 3, links). Als Projektionsebene bezeichne ich jene Ebene, die sich im Gesichtsfeld des Betrachters als Fenster zum Bildraum hin öffnet und in diesem Sinn eine Ebene mit rein optischem Charakter ist. Im perspektivischen System ist diese Ebene spätestens seit Alberti als Grundfläche einer Pyramide bestimmt, welche prinzipiell auf jede materielle Fläche mit beliebiger Oberflächenbeschaffenheit projiziert werden kann. Die Projektionseben ist in diesem Sinn eine rein systemische Ebene, die zwar nicht in Erscheinung tritt, jedoch für die Konstruktion perspektivischer Sichtbarkeit eine implizite Rolle spielt, da sie gewissermaßen das optische Lot eines Bildes bestimmt.

Das anamorphotische Darstellungsverfahren beruht nun darin, eine Rotation einzuführen, mit der eine Winkeldifferenz zwischen der Projektionsebene und der empirischen Bildfläche entsteht (Abb. 3, Mitte). Der Schnitt durch die Sehpyramide ist hier also nicht rektangulär realisiert, weshalb die Proportionen des Dargestellten aus der frontalen Sicht derart 
verzerrt erscheinen, dass das Dargestellte nicht mehr als solches erkennbar ist. Vielmehr zeigt sich die Bildfläche dann als kryptisch pigmentierte Fläche und erst wenn der Betrachter die geometrisch korrekte und seitliche Position vor dem Gemälde einnimmt, fügt sich das sichtbare Material zu einem Bild. Bei der Anamorphose handelt es sich so gesehen um ein kalkuliertes Spiel zwischen Deformation und Formation der Sichtbarkeit, die mit dem Eintreten bzw. Verlassen des korrekten Blickpunktes verbunden ist und die auf der misslingenden bzw. gelingenden Wahrnehmung einer perspektivisch generierten Darstellung basiert.

Die Anamorphose führt damit zunächst vor Augen, worin die gestalterische Funktion der Perspektive eigentlich liegt: Sie regelt zunächst die Beziehung zwischen einem materiellen Bildträger und einer räumlich dargestellten Bilderscheinung und setzt diese Beziehung wiederum in Beziehung zu einem situierten Blickpunkt - ein Verfahren, dass Ralph Ubl und Wolfram Pichler als »Formatierung« bezeichnen. Anamorphotische Darstellungen sind ein Beispiel dafür, wie diese Relationalität von Beziehungen eingesetzt werden können. Das Phänomen der Anamorphose belegt weiter, dass es bereits in der frühen Geschichte der Perspektive ein Bewusstsein für die systematische Unterscheidung von Projektionsebene und Bildfläche gibt, mithin dass die Suggestion der Identität des Bildes mit dem Schnitt durch die Sehpyramide, die in Abertis berühmter Fenstermetapher zum Ausdruck kommt, keineswegs zwingend ist, sondern nur eine unter vielen möglichen Verwendungsweisen der perspektivischen Projektion. ${ }^{18}$ Es mag sein, dass die Perspektivisten des 15. und 16. Jahrhunderts es vorzogen, die Projektionsebenen möglichst bruch- und nahtlos mit dem Träger zu verbinden, doch wie die Perspektivtraktate des 17. Jahrhundert belegen, ist schon bald eine Vielzahl raffinierter Varianten perspektivischer Konstruktion entstanden, mit denen die Differenz von Projektionsebene und Bildfläche gezielt eingesetzt wird. Weiter zeigt die Anamorphose, dass die rechtwinklige Frontalität zwischen Betrachter und Bild, genauer gesagt zwischen der Sichtachse als zentralem Sehstrahl und der materiellen Bildfläche, keine notwendige Voraussetzung perspektivischer Sichtbarkeit ist.

Die perspektivische Formatierung eines Bildträgers ermöglicht damit eine Vielzahl von Möglichkeiten, mit denen das Dargestellte und sein Bildraum auf den Betrachter im Realraum bezogen werden kann. Ihre Verwendung erlaubt also Modifikationen und ist nicht, wie vielfach angenommen, ein komplett reguliertes oder statisches Verfahren. Denn wenn die Grundlage der Anamorphose gerade im spannungsvollen Zusammenspiel zwischen projizierter

18 Vgl. dazu auch Patrick Maynard: Drawing Distinctions - The Varieties of Graphic Expression, Ithaca $2005,28 \mathrm{f}$. 
Sichtbarkeit und ihrer materiellen Voraussetzungen liegt, zeigt sich damit auch, dass die materielle Bildfläche ein zentraler Bestandteil perspektivischer Sichtbarkeit ist, deren Modifikation (etwa durch Rotation) das Sichtbare empfindlich verändert. Die bildliche Sichtbarkeit siegt also nicht auf Kosten des Bildträgers und seiner Fläche, sondern tritt mit ihm in ein Wechselspiel, das die Grundlage ikonischer Sichtbarkeit bildet. Die materielle Bildfläche und ihre jeweils spezifische Beziehung zur projizierten Sichtbarkeit muss folglich als integratives Element der perspektivischen Disposition begriffen werden.

In der Anamorphose kommen also die komplexen Beziehungen zwischen projizierter Sichtbarkeit, materiellem Träger und Betrachterstandpunkt zum Ausdruck. Inwiefern handelt es sich dabei aber um eine Kritik? Welche Bedeutung hätte der Begriff der \Kritik` in diesem Zusammenhang überhaupt und wie kann man diesen mit dem modernistischen Paradigma selbstreflexiver Kritik und damit einem Diskurs in Beziehung setzen, der die Perspektive als Problem bestimmt?

Wie beschrieben liegt der primäre Effekt der anamorphotischen Darstellung im dynamischen Wechselspiel zwischen Formierung und Deformation des Sichtbaren. Dieses Wechselspiel zwischen Formierung und Deformation perspektivischer Sichtbarkeit kann nun verschieden interpretiert werden. Man könnte entweder zum Schluss kommen, dass es sich letztlich um eine Affirmation der Perspektive handelt, da unter bestimmten Voraussetzungen das Gelingen des perspektivischen Bildmodells erlebbar gemacht wird, womit die Theorie perspektivischen Sehens verifiziert ist. Oder aber man kann die anamorphotische Deformation zum Anlass nehmen, um den Trugcharakter der Perspektive hervorzuheben, oder gar, wie Descartes es tut, um die sinnliche Wahrnehmung schlechthin in Frage zu stellen.

Mir scheint, dass beide Positionen, die man verkürzt als Realismus bzw. Skeptizismus bezeichnen könnte, der eigentlichen Leistung der Anamorphose nicht gerecht werden, denn die Anamorphose stellt weder die perspektivische Theorie unter Beweis, noch ist sie eine Falsifizierung derselben. Vielmehr scheint es mir angemessen, von einer Explikation der notwendigen Bedingungen zu sprechen, unter denen perspektivisch konstruierte Sichtbarkeit stattfinden kann. Der Betrachter einer anamorphotischen Darstellung in unserem Sinne erlebt vor allem das vorausgesetzte Verhältnis zwischen dem bildlich Dargestellten und dem Bildträger sowie dem Verhältnis dieses Verhältnisses zu seinem eigenen Standpunkt. Es sind also gerade die konstitutiven Beziehungen von Darstellung und Dargestelltem und weiter die Beziehung dieser Beziehung zum Betrachter, die als solche bemerkbar werden. 
Für die Frage nach der Kritik spielt das skeptische Moment der Anamorphose jedoch eine entscheidende Rolle: Die Verzerrung des Sichtbaren, die Descartes bekanntlich zum Anlass nimmt um die Gewissheit der sinnlichen Wahrnehmung zu problematisieren, beruht buchstäblich auf dem Austritt aus einem perspektivischen System. Was von einem Blickpunkt aus sichtbar ist, deformiert sich zur Unkenntlichkeit, wenn es von einem anderen Blickpunkt aus gesehen wird, der nicht systematischer Teil des perspektivischen Dispositivs ist. ${ }^{19}$ Der mit diesem Austritt gleichsam empirisch gewordene Blick auf eine nunmehr bloß kryptisch pigmentierte Fläche ist nicht mehr in der Lage, einen Bildraum oder ein Dargestelltes, ja überhaupt ein Bild wahrzunehmen. Wenn man also die Anamorphose als Kritik der Perspektive verstehen möchte, muss man verstehen, dass die Anamorphose diese Kritik immer nur auf Kosten der Sichtbarkeit des Bildes formulieren kann, denn ihr zentraler Effekt beruht buchstäblich auf dem Austritt aus dem perspektivischen System. Die anamorphotische Kritik der Perspektive ist demnach wesentlich transzendent, sie ist Kritik von außen, denn sie zwingt den Betrachter dazu, einen systemexternen Standpunkt einzunehmen, der die perspektivische Sichtbarkeit negiert. Die anamorphotische Kritik der Perspektive operiert demnach an den Grenzen des perspektivischen Dispositivs, dessen Bedingungen nur dadurch freigelegt werden, indem sie überschritten werden.

\section{Die Perspektive wiederentdecken}

Gemessen an der greenbergschen Konzeption einer kritischen Moderne lässt die Anamorphose also eine selbstreflexive Kritik im eigentlichen Sinn vermissen. Ich erinnere nochmals daran, dass Greenberg die Perspektive auf der Basis seines kunsttheoretischen Programms in Frage stellt, ein Programm, das nicht an einer Äquivalenzbeziehung zwischen Perspektivbild und einem vermeintlichen Wahrnehmungsbild interessiert ist, sondern auf einer Logik aufbaut, die ich selbstreflexiven Kritizismus nenne. Im weiteren werde ich der Frage nachgehen, ob und wie die perspektivische Form diesem selbstreflexiven Kritizismus gerecht werden kann, denn wie die Rekonstruktion von Panofskys Argumentation gezeigt hat, ist die Perspektive als epistemologisches Modell mit der Kritik der Perspektive selbst verwoben. Mit anderen Worten

19 Zwar kann man dafür argumentieren, dass das Verlassen des perspektivischen Standpunktes kalkuliert ist und daher zum System der Anamorphose gehört, dieses Kalkül ist aber nicht Teil der Perspektive als solcher, wie Dieter Mersch annimmt: Abbild und Zerrbild - Zur Konstruktion von Rationalität und Irrationalität in frühneuzeitlichen Darstellungsweisen, in: Theatrum Scientiarum, Bd. 2: Instrumente in Kunst und Wissenschaft, hg. von Helmar Schramm, Ludger Schwarte und Jan Lazardzig, Berlin/New York 2006, 22-39. 
bedeutet das, dass die in diesem Fall theoretischen Mittel der Perspektive zur Kritik derselben eingesetzt werden können. Im Folgenden werde ich nach der künstlerischen Tragweite dieses selbstkritischen Potentials fragen.

In der jüngeren Kunstproduktion lässt sich ein ernsthaftes Interesse an Verfahren der perspektivischen Bildgebung nachweisen, welche sich jene kritische Kapazität perspektivischer Form zu eigen macht, die in der Netzhautbild-Kritik sowie in Panofskys historisierender Kritik der Perspektive latent bemerkbar wird. Dass heute eine künstlerische Arbeit mit der Perspektive gerade im Zusammenhang mit einer kritischen Lektüre des modernistischen Erbes besonders produktiv sein kann, zeigt die Malerei der US-Amerikanerin Rebecca H. Quaytman. Sie leistet eine so komplexe wie intelligente Rehabilitierung der perspektivischen Darstellungsform, und zwar unter Berücksichtigung jener historischen Diskurse, mit denen die Perspektive im 20. Jahrhundert verunglimpft wurde. ${ }^{20}$ Die zwei Gemälde aus der Serie The Sun (2001) bestehen, wie alle ihre Gemälde, aus mehreren Zentimeter dicken Sperrholzplatten, deren Oberflächen auffällig glatt und im Siebdruckverfahren bearbeitet wurden (Abb. 4 und 5). Der Vergleich der beiden Gemälde zeigt deutlich, wie die fotografische Vorlage nicht bloß bildinterne perspektivische Verkürzungen aufweist, sondern in einem Gemälde abermals einer Perspektivierung unterworfen ist. Die fotografische Darstellung des Inneren eines Zugwagons wirkt, ${ }^{21}$ als wäre sie scharnierartig ins Gemäldeinnere geklappt. Sie fluchtet in eine leere monochrome Farbfläche, die zugleich den Malgrund für den Siebdruck bildet.

Das Ergebnis ist eine besondere perspektivische Konfiguration des Gemäldes, die auf Seiten des Betrachters sowie des Gemäldes starke Konsequenzen mit sich bringt. Einerseits ist die resultierende Form im Hinblick auf den sie tragenden und dabei distanzierten Blickpunkt zu verstehen. Unabhängig von der Frage, ob dieser Blickpunkt möglicherweise Kennzeichen neuzeitlicher Subjektivität aufweist und wie man eine Verbindung zwischen Perspektivismus und Subjektivismus plausibel machen kann, muss festgehalten werden, dass die im Bild implizite Adressierung des Betrachters von ganz bestimmter Art ist. Denn in einem sind sich alle Theoretiker einig: Die perspektivische Form ist wesentlich eine standpunktbezogene Form, ganz ungeachtet dessen, wie dieser Standpunkt begriffen bzw. besetzt wird. Dieser implizite Standpunkt ist in seiner Qualität also zunächst indifferent, es handelt sich um einen geometrisch

\footnotetext{
20 Zum Zusammenhang von Perspektive und Modernismus im Werk von Quaytman siehe etwa Rebecca H. Quaytman: Allegorical Decoys, Gent 2008, dort S. 13-15 und S. 23; sowie das Gespräch mit Steel Stillman: Interview with Rebecca H. Quaytman, in: Art in America 6 (2010), 87-95, hier 93.

21 Die Ikonografie der beiden Gemälde verweist primär auf die familiäre Herkunft der Künstlerin, deren Grossvater aus dem polnischen Lodz emigriert ist. Die Fotografie ist bei einer Zugfahrt von Quaytman nach Lodz entstanden. Rebecca H. Quaytman: Spine, Berlin 2011, 7.
} 
formalisierten Punkt. ${ }^{22}$ Nun hat diese reduktionistische Formalisierung des Betrachters wiederum jene Kritiker auf den Plan gerufen, die betonen, dass die Perspektive die sinnliche Leiblichkeit zuungunsten des Bildes sowie zuungunsten des Betrachters übergehe. Indem die Perspektive die Anlage des Bildes konsequent geometrisch systematisiere und auf einen formalisierten sowie fixierten Blickpunkt hin ausrichte, sei sie, wie Maurice Merleau-Ponty sagt, ein »malin dispositif«. ${ }^{23}$ Unklar bleibt, weshalb diese Abschwächung der affektiven Koppelung des Leibes zum Bild unbedingt defizitär sein muss. Zeigt Quaytmans Verwendung der Perspektive nicht, dass man eben jene Eigenschaften der perspektivischen Form, die unter phänomenologischen Vorzeichen als defizitär eingestuft werden, gezielt nutzen und damit künstlerisch fruchtbar machen kann? Nicht zufällig entspricht der Entaffizierung des formalisierten Blickpunktes die anonyme fotomechanische Bildproduktion sowie der Verzicht auf Farbe, was schließlich zu einer derart distanzierten Adressierung des Betrachters führt, dass man mit Martin Jay geradezu von einer »De-Erotisierung« sprechen kann. ${ }^{24}$ Ihre Verwendung der Perspektive lässt sich in diesem Sinn als eine Strategie begreifen, die sich einen von der modernen Bildtheorie immer wieder kritisierten Aspekt der perspektivischen Formgebung zu eigen macht, um damit unter veränderten historischen Bedingungen bestimmte Ziele zu verfolgen.

Die Konsequenzen der perspektivischen Konfiguration zeigen sich aber auch auf der gegenüberliegenden Seite des Blickpunktes. Denn die distanzierte Beziehung des Betrachters zum Gemälde bedeutet nicht, dass die Materialisierung des Bildes zu einem vernachlässigbaren Faktor wird. Vielmehr liegt die bemerkenswerte Leistung des Gemäldes gerade im besonderen Verhältnis zwischen Bildraumorganisation und Flächenorganisation, d.h. der spezifischen Inbezugnahme der beiden. Man bedenke zunächst, dass die Perspektive als raumgebendes Mittel funktioniert, indem sie die Bildelemente formal so anordnet, dass die systematisch nachvollziehbare Illusion einer dreidimensionalen Bildtiefe entsteht. Diesen Raum, seine kontinuierliche Tiefe und die damit generierte Disposition des Blickpunkts, setzt sie zugleich in explizite Beziehung zu einer materiellen Fläche. Diese letzte Beziehung wird in aller Regel wenig bedacht, was auch daran liegt, dass die gängige Verwendung der perspektivischen Bildproduktion darum bemüht ist, sie unsichtbar zu machen, indem sie eine Identität zwischen systemischer Projektionsfläche und materieller Bildfläche suggeriert. Die verwendete Perspektivierung unterbricht nun diese nahtlose Überblendung des Bildträgers durch die

22 Der formale Charakter dieses Punktes hat dazu veranlasst, diesen in unterschiedlicher Weise zu besetzen. Dazu etwa Brian Rotman: Die Null und das Nichts - Eine Semiotik des Nullpunkts, Berlin 2000.

$23 \quad$ Maurice Merleau-Ponty: L'oil et l'esprit, Paris 1964, 20.

24 Martin Jay: Scopic Regimes of Modernity, in: Vision and Visuality, hg. von Hal Foster, Seattle 1988, 323 , hier 8 . 
Projektion von drei Bildrändern, wodurch nicht nur die drohende infinite Regression der Selbstdarstellung unterbunden wird, sondern auch das materielle Substrat des Bildes, sein Format und seine Flächigkeit thematisch werden. ${ }^{25}$ Die vorliegende Perspektivierung verweist so auf die allzu oft vergessene Gegebenheit, dass das opake Element der materiellen Bildfläche ein fundamentaler Bestandteil der perspektivischen Form ist, mithin dass die Perspektive nicht bloß die vielfach betonte Beziehung zwischen Blickpunkt und Fluchtpunkt herstellt, sondern diese Beziehung abermals mit einer materiellen Ebene in Beziehung setzt. Indem die im Gemälde hergestellte Metaperspektive die Illusion einer kontinuierlichen Identität von perspektivischer Projektion und Bildfläche unterbricht, artikuliert sie also eine Differenz, die dem perspektivischen Bildmodell wesentlich ist, jedoch kaum je sichtbar gemacht wird. Die perspektivische Form ist hier als jenes »Medium« begriffen, das, wie Michael Fried sagt, eine Wechselbeziehung herstellt zwischen dem materiellen Träger und demjenigen, was in und mit diesem Träger erscheint. ${ }^{26}$

Die Arbeit Quaytmans macht in diese Sinne deutlich, dass die Bildfläche ein Teilelement der perspektivischen Disposition ist und dass man ihrer Rolle nicht gerecht wird, wenn man sie als zu negierende Materialität, als bloß transitorische Größe denkt. Vielmehr ist sie Teil einer Ordnung, deren Funktion gerade darin besteht, mit einer materiellen Ebene eine räumliche Erscheinung zu generieren und diese mit der Betrachterposition auf eine jeweils bestimmte Weise in Beziehung zu setzen, um unter gewissen Bedingungen abermals bestimmte Absichten $\mathrm{zu}$ verfolgen. Begreift man die Perspektive in diesem Sinn als ein Instrument, das zu bestimmten Zielen eingesetzt werden kann, verdeutlicht sich auch ihre mögliche Rolle in der Kunst der Gegenwart. Denn im Hinblick auf die Perspektive und ihre moderne Geschichte sehen sich die Künstler der Gegenwart einigen Problemen ausgesetzt, die ihr die modernistische Malereitradition zumutet. Quaytman, deren Vater Maler der New Yorker Nachkriegsavantgarde war, ist mit diesen Diskursen überaus vertraut. Ihre Entscheidung Malerei zu betreiben ist auch eine bewusste Entscheidung, sich in ein historisch komplexes, um nicht zu sagen belastetes Feld einzuschreiben. Die Perspektive spielt dabei eine entscheidende Rolle, bildet sie als illusionistisches Gestaltungsmittel doch gerade eine Zielscheibe des modernistischen Ikonoklasmus. Die historische Kritik der Perspektive bildet für die Generation

\footnotetext{
25 Jüngst hat Johannes Grave darauf aufmerksam gemacht, dass bereits die Malerei des Quattrocento die Randzonen perspektivischer Bilder als produktive Irritationsmomente einsetzt. Johannes Grave: Architekturen des Sehens, Paderborn 2015.

$26 \quad »[\ldots]$ shape as a medium within which choices about both literal and depicted shapes are made, and made mutually responsive". Michael Fried: Shape as Form - Frank Stella's Irregular Polygons (1966), in: Art and Objecthood, Chicago/London 1998, 77-99, 77.
} 
junger Malerinnen und Maler wie Quaytman also eine schwierige und zugleich delikate Ausgangslage. Denn einerseits erbt Quaytman das modernistische Misstrauen gegenüber der projektiven Geometrie: »The evacuation of that system left me as a young painter, with an intense suspicion and immediate distrust of perspective. ${ }^{27}$ Andererseits schwebt ihr die Möglichkeit einer dialektischen Aussöhnung vor: »The edges, the picture plane, and the object of the painting could still be experienced in tandem with the illusionistic space of the picture. $\ll^{28}$

Ich argumentiere dafür, dass diese Wiederentdeckung der Perspektive eine Konfiguration des Gemäldes vollzieht, die vor dem Hintergrund der modernistischen Malereitradition als Kritik und letztlich als Emanzipation von einem historischen Paradigma der Malerei begriffen werden muss. Ich kann diese Kritik anhand dreier Aspekte beschreiben. Da ist zunächst der rationale Charakter der perspektivischen Konstruktion, der einer originellen, inspirierten oder sonst auratisierten Einschreibung des Malersubjekts auf der Bildfläche widerspricht. Die anonyme geometrische Konstruktion und ihre prinzipielle Reproduzierbarkeit stehen in scharfem Gegensatz zur Idee einer authentischen Autorschaft und findet in der ebenso anonymen fotomechanischen Produktion des Gemäldes ihre Entsprechung. Zweitens findet eine Umdeutung der Bildfläche statt, die durch das projektivgeometrische Verfahren mehr als neutrale Projektionsfläche denn als signifikante Materialität bestimmt wird. Dieser Umstand wird besonders durch die Beschaffenheit der Bildfläche unterstrichen, deren Glätte und Homogenität der modernen Idee des Gemäldes als einer fakturierten Einschreibungsfläche entgegensteht und damit mit der intensivierten Beziehung des Trägermaterials zur Bilderscheinung bricht. Drittens ist der radikale Verzicht auf Farbe auffällig, der im Sinn eines rational operierenden disegnos als Loslösung vom modernen Kolorismus interpretiert werden kann.

Die Rationalität und Anonymität, die durch die perspektivgeometrische und mechanische Entkörperlichung des Gemäldes erzeugt wird, hängt wesentlich mit der vom Werk explizierten Autorschaft zusammen, was etwa auch durch Quaytmans konsequente Reduktion aller Bildanschriften und -legenden auf die bloßen Initialen der Künstlerin betont wird. Quaytmans Projekt nutzt also die Perspektive, um sich gegen eine von der Moderne tradierten Konzeption von Malerei zu wenden, die das Bild primär als stofflich signifikantes Produkt eines körperlich agierenden Künstlersubjekts begreift und die Bildfläche als Ort der Oberflächeneinschreibung. Die Perspektive, ihre Eigenschaften und bildtheoretischen Implikationen werden so gesehen reaktiviert, um Kritik $\mathrm{zu}$ üben an einem seinerseits

27 Email-Konversation zwischen Apsara Di Quinzo und Rebecca Quaytman, publiziert anlässlich der Ausstellung New Work 22.10.-16.01.2011 im San Francisco Museum of Modern Art.

28 Ebd. 
historischen Bildmodell. Die Perspektive zeigt sich damit als eine Art Vehikel der Kritik, einer Kritik die man am besten als diskursiv beschreibt, denn sie operiert wesentlich mit einer dialektischen Wende, die ein Modell des Bildes durch ein anderes Modell ersetzt. Unter bestimmten historischen Voraussetzungen auf ein besonderes historisches Modell der Sichtbarkeitserzeugung zurückzugreifen, kann demnach kritische Effekte erzeugen. Es stellt sich aber die Frage, ob sich das kritische Potential der Perspektive in dieser diskursiven Kritik erschöpft. Wie steht es um die Selbstkritik im engeren Sinn? Gibt es eine genuin perspektivische Kritik, die sich mit den Maßstäben des modernistischen Reflexionsparadigmas messen kann, einem Paradigma, das die Kritik eines Systems als Effekt desselben Systems festlegt?

Man könnte zum naheliegenden Fehlurteil gelangen, es handle sich bei der seltsam verzerrten Sichtbarkeit in Quaytmans Gemälde um eine anamorphotische Darstellung. Ich habe jedoch bereits erläutert, wie die Anamorphose das Eintreten bzw. Verlassen des korrekten Blickpunktes vor dem Bild einsetzt, um damit die Formierung bzw. Deformation des perspektivisch Dargestellten zu bewirken. Mit anderen Worten, der Betrachter tritt aus dem perspektivischen System aus, was die anamorphotische Kritik der Perspektive als eine Kritik von außen kennzeichnet. Quaytmans Verwendung der Perspektive unterscheidet sich nun maßgeblich von dieser anamorphotischen Verwendung, was etwa daran erkennbar wird, dass die perspektivische Verzerrung der fotografischen Darstellung sich in keinem Fall - und erst recht nicht durch Bewegung vor dem Gemälde - auflösen lässt. Dies liegt an der spezifischen räumlichen Konstruktion von Quaytmans Darstellung, welche eine eigentümliche Verdoppelung der Perspektive herbeiführt. Wie die schematische Rekonstruktion des Grundrisses (Abb. 3, rechts) zeigt, ist die perspektivische Formierung des fotografisch Dargestellten einer weiteren Perspektivierung unterworfen, indem ein zweites Perspektivsystem mit einem zweiten Augpunkt, einem zweiten Fluchtpunkt und einer zweiten Projektionsebene eingeführt wird. Die hier vorliegende Rotation ist also keine zwischen Projektionsebene und empirischer Bildfläche, wie bei der Anamorphose, sondern eine zwischen einer ersten und einer zweiten Projektionsebene, die dabei eine gemeinsame Horizonthöhe teilen. Anstelle einer Deformation der perspektivischen Darstellung findet so eine Perspektivierung der perspektivischen Form statt, wodurch ebendiese Form nicht dieselbe bleibt. Die damit erzeugte Metaperspektive macht nicht etwa als Negation, Bruch oder Störung auf die perspektivische Form und deren Konstitution aufmerksam, vielmehr ermöglicht sie die Explikation perspektivischer Formkonstitution mit deren eigenen Mitteln. 
Der aus perspektivtheoretischer sowie modernistischer Sicht interessante Aspekt dieser Metaperspektive liegt denn auch genau in dieser Möglichkeit, die Kritik der Perspektive nicht auf Kosten der perspektivischen Sichtbarkeit zu formulieren. Es ist hier die Perspektive selbst, die ihre Kritik ermöglicht. Ihr Formgesetz wird gerade nicht durch Negation desselben expliziert, sondern mit dessen eigenen Mitteln. Folglich handelt es sich bei der hier zum Tragen kommenden Kritik erstens um eine Reflexion im engeren Sinne, d.h. eine Bewegung der Immanenz, in der das Subjekt der Reflexion mit dem Objekt der Reflexion identisch ist. Und sie ist zweitens in der Lage, die für eine Kritik nötige Distanz von eben jenem System, das sie selbst ist, zu erzeugen. Die perspektivische Form ist in der Lage von sich selbst zu differieren, um zugleich zu sich zurückzukehren. Erst diese Selbstdifferenz, in welcher Transzendenz und Immanenz zusammengehen, macht es möglich, dass sich Form zugleich kritisch und reflexiv auf sich selbst beziehen kann.

Es scheint, als sei das populäre modernistische Narrativ, das die perspektivische Transparenz gegen kritische Selbstreflexion ausspielt, weitgehend blind für das kritische Vermögen der Perspektive. Dies liegt vor allem daran, dass sich sein Reflexionsparadigma ab einem gewissen Zeitpunkt einseitig auf die materielle Fundierung des Bildes und ein damit verbundenen Paradigma von Malerei verengt hat. Dieser Materialismus ist deswegen besonders bedauerlich, weil es ihm nicht gelingt, die perspektivischen Reflexionsleistungen geometrisch-räumlicher Konstruktionen zu verstehen. Die genaue Rekonstruktion dieser Leistungen hilft nicht nur, unser Verständnis von Reflexion und Kritik zu präzisieren, sondern eröffnet auch neue Perspektiven auf den Modernismus. 


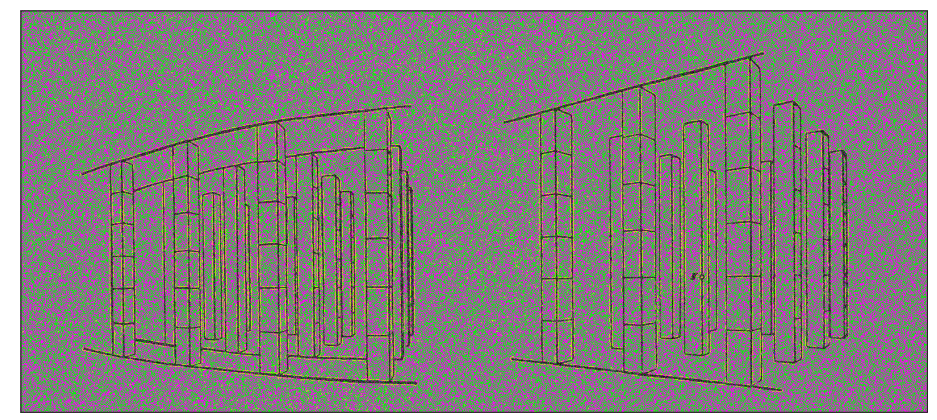

Abb. 1

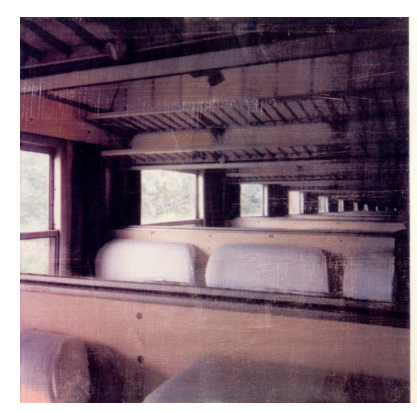

Abb. 2

Abb. 3

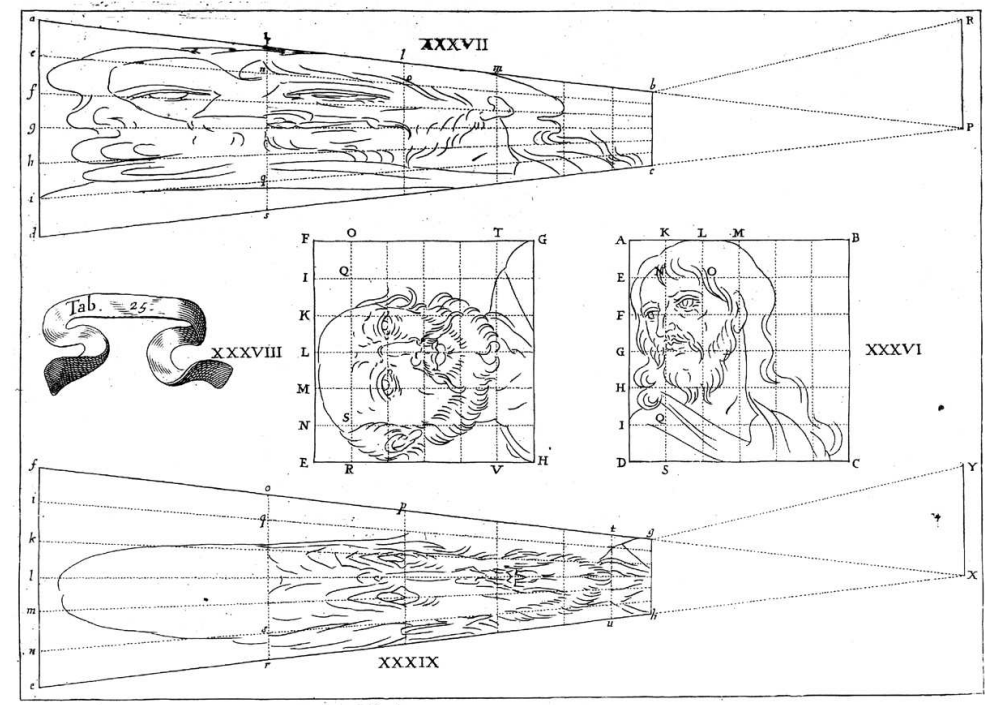

Abb. 4
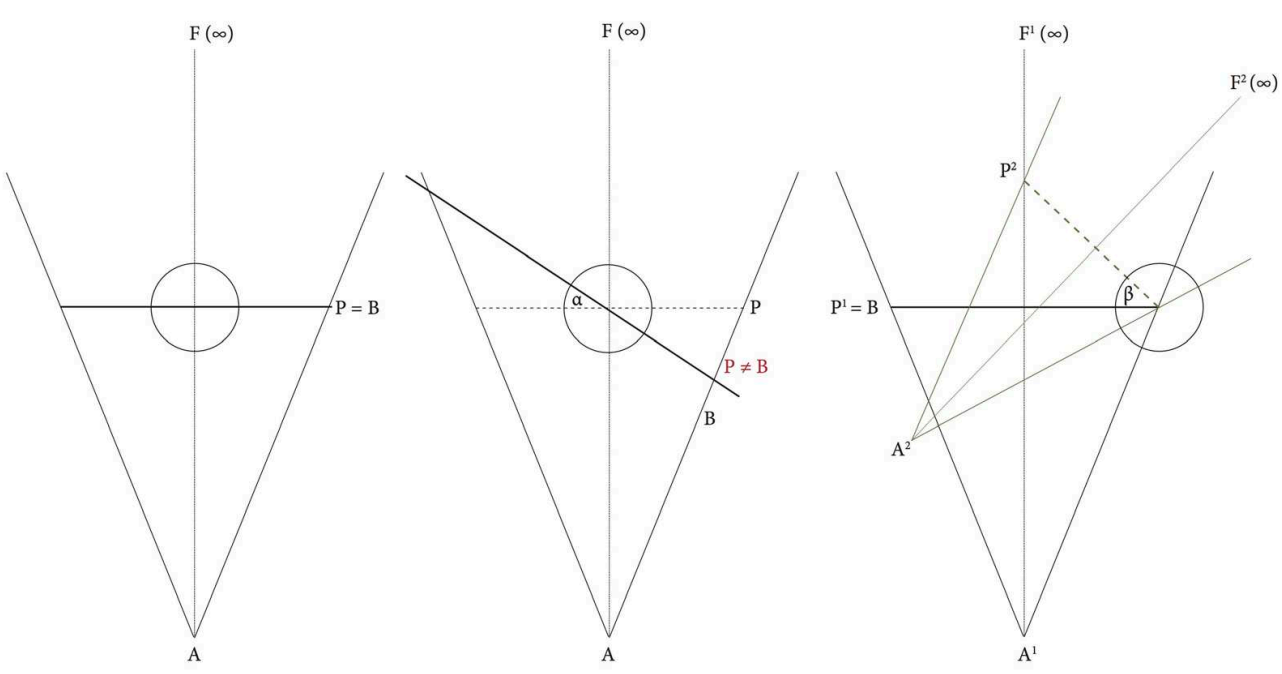

Abb. 5 